\section{Validade das medidas auto-referidas de peso e estatura para o diagnóstico do estado nutricional de adolescentes}

\section{Validity of self-reported weight and height for adolescent nutritional status diagnosis}

José Cazuza de Farias Júnior ${ }^{1}$

1 Programa de Pós-graduação em Educação Física. Centro de Desportos. Universidade Federal de Santa Catarina.Trindade Florianópolis, SC, Brasil. CEP 88.040-900. E-mail: jcazuzajr@hotmail.com

\section{Abstract}

Objectives: analyze self-reported weight and height measurements to diagnose adolescents nutritional state.

Methods: eight hundred and sixty seven adolescents (433 boys and 434 girls) of the municipality of Florianópolis, Santa Catarina aged between 15 and 18 years old $(16.2 \pm 1.08)$. Initially the adolescents would report their weight and height; following the respective measurements would be taken by the researchers. The Body Mass Index (BMI) was established based on the self-reported and taken measurements.

Results: when reporting their anthropometric measurements, in average, adolescents would underestimate their weight in $0.5 \mathrm{~kg}$, overestimated their height in $0.42 \mathrm{~cm}$ and the BMI based on these measurements were underestimated in $0.3 \mathrm{~kg} / \mathrm{m} .{ }^{2}$. Self-reported measurements had high correlation with the measurements taken (intra-class correlation coefficient - ICC - 0.91 to 0.96). Validity rates of BMI determined from the self-reported measurements varied between $60 \%$ and $79.1 \%$ for sensitivity, $98.4 \%$ to $99.2 \%$ for specificity, $89.8 \%$ and $90 \%$ for positive predictive value. Overweight/obesity was underestimated in $2.6 \%$ when determined from the selfreported measurements $(p=0.18)$.

Conclusions: weight and height self-reported measurements are valid as an approximation of the measurements taken and can be used when assessing adolescents nutritional state based on BMI in epidemiological studies.

Key words Overweight, Obesity, Adolescence

\section{Resumo}

Objetivos: analisar a validade das medidas autoreferidas de peso e estatura para o diagnóstico do estado nutricional de adolescentes.

Métodos: fizeram parte do estudo 867 adolescentes (433 rapazes e 434 moças), do município de Florianópolis, Santa Catarina, com idades entre 15 e 18 anos (16,2 1,08). Inicialmente, os adolescentes referiram o peso e a estatura; na seqüencia, as respectivas medidas foram aferidas pelos pesquisadores. Determinou-se o índice de massa corporal (IMC) com base nas medidas referidas e aferidas.

Resultados: ao referirem suas medidas antropométricas, em média, os adolescentes subestimaram o peso em $0,5 \mathrm{~kg}$, superestimaram a estatura em $0,42 \mathrm{~cm}$, e o IMC baseado nessas medidas foi subestimado em $0,3 \mathrm{~kg} / \mathrm{m}^{2}$. As medidas auto-referidas apresentaram concordância elevada com as medidas aferidas (coeficiente de correlação intraclasse - CCIC $=0,91$ a 0,96). Os índices de validade relativa do IMC, determinado a partir das medidas referidas, oscilaram entre $60 \%$ e 79,1\% para sensibilidade, 98,4\% a 99,2\% para especificidade, $89,8 \%$ e $90 \%$ para o valor preditivo positivo. A prevalência de sobrepeso/obesidade foi subestimada em 2,6\% quando determinada a partir das medidas referidas $(p=0,18)$.

Conclusões: as medidas auto-referidas de peso e estatura representam medidas válidas como forma de aproximação aos valores mensurados, podendo ser utilizadas na avaliação do estado nutricional de adolescentes a partir do IMC, em estudos epidemiológicos.

Palavras-chave Sobrepeso, Obesidade, Adolescente 
Introdução

A monitoração dos índices de sobrepeso/obesidade em crianças e adolescentes passou a representar uma das principais ações, na área de saúde pública, tendo em vista o rápido crescimento na prevalência de sobrepeso/obesidade e os problemas de saúde associados. ${ }^{1,2}$ Nesse sentido, o índice de massa corporal (IMC), determinado a partir de medidas autoreferidas de peso e estatura, em substituição às medidas mensuradas, passou a ser freqüentemente utilizado em levantamentos epidemiológicos que objetivam avaliar o estado nutricional de adolescentes. 3,4

Os resultados dos estudos que procuraram examinar a validade das medidas referidas de peso, estatura e do IMC obtido a partir dessas medidas têm descrito que, embora estejam altamente correlacionadas com as medidas aferidas, $5-7$ o peso autorelatado pelos adolescentes tende a ser menor do que a medida aferida, principalmente nas moças. 8,9 Para a estatura, os resultados têm sido discordantes. Enquanto alguns estudos descrevem que os adolescentes tendem a subestimar a estatura referida, 6,10 outros descrevem tendência para os adolescentes superestimarem.5,9,11 Em decorrência das discrepâncias entre as medidas referidas e aferidas, a prevalência de sobrepeso/obesidade determinada a partir do IMC, baseado em medidas referidas, tem sido subestimada em até $15 \%$, em comparação àquela determinada a partir do IMC de medidas aferidas. $6,8,9$

Diferenças quanto ao delineamento do estudo, características da amostra, análises estatísticas utilizadas e a influência de aspectos socioculturais, contribuem para as divergências apresentadas entre os estudos de validade das medidas auto-referidas por adolescentes. Portanto, a transferência de informações sobre a validade dessas medidas entre diferentes grupos populacionais, principalmente pertencendo a regiões geográficas distintas, fica comprometida, e deve ser evitada.

No Brasil, informações sobre a validade das medidas auto-referidas de peso e estatura são provenientes de investigações desenvolvidas em adultos, 12,13 de modo que ainda não se sabe com que precisão essas medidas são auto-referidas pelos adolescentes brasileiros. Nesse sentido, este estudo analisou a validade das medidas auto-referidas de peso e estatura para o diagnóstico do estado nutricional de adolescentes de ambos os sexos, do município de Florianópolis, Santa Catarina.

\section{Métodos}

No desenvolvimento do estudo, foram utilizadas as informações coletadas em um estudo transversal de base escolar "Estilo de Vida de Escolares do Município de Florianópolis - SC", realizado entre maio e agosto de 2001, com escolares do ensino médio do município de Florianópolis, Santa Catarina, localizado na região Sul do Brasil.

No processo amostral, adotou-se uma seqüência de duas etapas. Inicialmente foram selecionadas, aleatoriamente, 21 escolas de ensino médio (14 públicas e 7 privadas), das 98 existentes no município de Florianópolis. Na seqüência, em cada escola selecionada, após autorização da direção da escola, selecionou-se aleatoriamente, uma quantidade de turmas suficiente para se alcançar o número de sujeitos que garantisse a representatividade em relação à população de escolares do ensino médio do município, considerando a característica da escola (pública e privada) e a série de ensino (primeira a terceira). Todos os escolares da turma sorteada, que estavam na sala de aula no dia da coleta, foram considerados elegíveis para participar do estudo, mediante autorização prévia.

Dentre os 1117 escolares (parâmetros de amostragem: $60 \%$ de inatividade física, intervalo de confiança (IC95\%), erro estimado de 3\%, acréscimo de $10 \%$ como possível índice de perda), foram excluídos 102 escolares que não informaram o sexo e/ou idade $(9,2 \%), 96$ que não referiram o peso e/ou estatura $(8,6 \%)$, e 52 que se recusaram a realizar tais medidas (4,6\%). Em função disso, para fins deste estudo, foram elegíveis para análise 867 adolescentes, com idades entre 15 e 18 anos (Tabela 1).

$\mathrm{Na}$ coleta de dados, uma equipe composta por quatro pesquisadores, previamente treinados, aplicou um questionário em sala de aula, que requeria 25 a 30 minutos para seu preenchimento pelos escolares.

\section{Tabela 1}

Sujeitos analisados, distribuídos por idade e sexo. Florianópolis, Santa Catarina, 2001.

\begin{tabular}{lrrr}
\hline Idade (anos) & Rapazes & Moças & Total \\
\hline 15 & 116 & 139 & 255 \\
16 & 151 & 136 & 287 \\
17 & 98 & 103 & 201 \\
18 & 68 & 56 & 124 \\
Total & 433 & 434 & 867 \\
\hline
\end{tabular}


Além de fornecer informações sobre aspectos demográficos (sexo, idade cronológica), comportamentos relacionados à saúde (nível de prática da atividade física, hábitos alimentares, fumo e bebidas alcoólicas - variáveis não analisadas nesse estudo), os adolescentes foram estimulados a auto-relatarem o peso $(\mathrm{kg})$ e a estatura $(\mathrm{cm})$ e, na seqüência, as respectivas medidas foram mensuradas. Os participantes não eram informados sobre o objetivo de comparação entre os dados referidos e aferidos no momento do preenchimento do questionário.

O peso foi aferido mediante a utilização de uma balança eletrônica (Filizola) com precisão de $100 \mathrm{~g}$, estando o adolescente posicionado no centro da mesma, de costas para o indicador de medida, com roupas leves e descalço. A medida de estatura foi efetuada com o auxílio de uma fita métrica de material não elástico, com precisão de um $\mathrm{cm}$, fixada à parede em superfície de apoio plana. $\mathrm{O}$ adolescente estava na posição ereta, sem sapatos, braços estendidos ao longo do corpo, em apnéia respiratória e a cabeça posicionada sobre o plano de Frankfurt. Na aferição, utilizou-se um esquadro de madeira posicionado rente a cabeça. Ambas as medidas foram realizadas por um único pesquisador, seguindo à padronização descrita por Gordon et al. 14

$\mathrm{O}$ índice de massa corporal (IMC) foi determinado a partir da razão entre o peso $(\mathrm{kg})$ e a estatura $\left(\mathrm{m}^{2}\right)\left(\mathrm{IMC}=\right.$ peso $[\mathrm{kg}] /$ estatura $\left.\left[\mathrm{m}^{2}\right]\right)$, com base nas medidas auto-referidas pelos adolescentes e as medidas aferidas pelos pesquisadores.

Para cada adolescente se estimou o erro absoluto das medidas auto-referidas em relação às medidas aferidas (erro absoluto $=$ medida referida - medida aferida). Para efeito de interpretação, valores negativos indicam subestimação das médias autorelatadas pelos adolescentes, enquanto que valores positivos indicam superestimação.

Para comparar o erro médio do peso e estatura referidos, bem como do IMC determinados a partir dessas medidas em relação às medidas aferidas (medida referida - medida aferida), separadamente por sexo e idade, recorreu-se ao teste $t$ de Student pareado. Aplicou-se o teste t de Student não-pareado para detectar possíveis diferenças no erro médio das medidas referidas, entre rapazes e moças e entre as idades estudadas. A análise de variância com um fator foi utilizada para comparar o erro médio das medidas referidas entre as idades estudadas, separadamente por sexo. Determinou-se o coeficiente de correlação intraclasse (CCIC) para obtenção de uma medida sumária de concordância entre as informações auto-relatadas e aferidas.

Para descrever a validade relativa do IMC, baseado nas medidas referidas, foram calculados os índices de sensibilidade, especificidade e valor preditivo positivo (VPP), tendo como critério de referência o IMC, baseado nas medidas aferidas. Para tanto, os escolares foram classificados com e sem sobrepeso/obesidade, considerando os pontos de corte propostos por Cole et al. 15

Utilizou-se o teste de McNemar para comparar a prevalência de sobrepeso/obesidade estimada a partir do IMC, determinado a partir do peso e estatura auto-referidos e aferidos. Adotou-se um nível de significância $p<0,05$.

\section{Resultados}

A média de idade da amostra estudada foi $16,2 \pm$ 1,$08 ; 50,5 \%$ eram do sexo feminino; cerca de $70 \%$ estudavam em escolas da rede pública e $30 \%$ em escolas da rede particular de ensino. Em relação à região geográfica, 73,2\%, 17,2\% e 9,6\% dos escolares investigados residiam na região central, continental e periférica da ilha (município de Florianópolis), respectivamente.

Na Tabela 2, estão descritas as médias de erro absoluto do peso, estatura e do IMC determinado com base nessas medidas. Em média, os rapazes estimaram o peso em - $0,26 \mathrm{~kg}(p=0,89)$ e as moças em $-0,87 \mathrm{~kg}(p=0,02)$. O erro médio do peso referido (medida referida - medida aferida) foi estatisticamente mais elevado nas moças do que nos rapazes $(-0,26$ vs $-0,87 ; p<0,01)$. Não foram observadas diferenças significativas, no erro médio do peso referido entre as idades estudadas (rapazes $\mathrm{F}=2,33$; $p=0,07$ e moças $\mathrm{F}=0,92 ; p=0,42$ ). Menos de $1 \%$ dos rapazes e pouco mais de $2 \%$ das moças relataram o peso de forma correta (medida referida $=$ medida aferida). Cerca de dois terços (65\%) dos adolescentes (63\% dos rapazes e $67 \%$ das moças) relataram o peso com diferença de até $2 \mathrm{~kg} ; 88 \%$ dos rapazes e $89 \%$ das moças relataram com diferença de até $5 \mathrm{~kg} ; 11 \%$ dos rapazes e $10 \%$ das moças com diferença maior que $5 \mathrm{~kg}$ (dados não apresentados).

Os adolescentes, em média, referiram a estatura com valores superiores à medida real $(+0,08 \mathrm{~cm}$; rapazes; $p=0,52$ e moças $+0,92 \mathrm{~cm} ; p=0,01)$. Como observado em relação ao peso, os rapazes referem à estatura com maior precisão que as moças $(0,08$ vs 0,$92 ; p=0,03)$. O erro médio da estatura referida (medida referida - medida aferida) não apresentou diferenças estatísticas entre as idades estudadas (rapazes $\mathrm{F}=0,92 ; p=0,43$ e moças $\mathrm{F}=0,98 ; p$ $=0,40)$. 
Tabela 2

Médias e desvios-padrão dos erros das medidas do peso, estatura e índice de massa corporal (IMC) determinados a partir das medidas referidas. Florianópolis, Santa Catarina 2001.

\begin{tabular}{|c|c|c|c|c|c|c|}
\hline \multirow[t]{2}{*}{ Idade (anos) } & \multicolumn{2}{|c|}{ Peso (kg) } & \multicolumn{2}{|c|}{ Estatura $(\mathrm{cm})$} & \multicolumn{2}{|c|}{ IMC (kg/m²) } \\
\hline & Rapazes & Moças & Rapazes & Moças & Rapazes & Moças \\
\hline $15-18$ & $-0,26( \pm 3,0)$ & $-0,87( \pm 2,7)^{a, b}$ & $+0,08( \pm 2,7)$ & $+0,92( \pm 2,5) a, b$ & $-0,07( \pm 1,2)$ & $-0,56( \pm 1,3) a, b$ \\
\hline 15 & $-0,34( \pm 2,5)$ & $-0,95( \pm 3,0)^{b}$ & $-0,01( \pm 2,4)$ & $+0,74( \pm 1,9)^{b}$ & $-0,17( \pm 1,1)$ & $-0,52( \pm 1,3)^{b}$ \\
\hline 16 & $-0,33( \pm 3,4)$ & $-1,11( \pm 2,8)^{b}$ & $-0,28( \pm 2,8)$ & $+0,84( \pm 2,7)^{b}$ & $-0,13( \pm 1,3)$ & $-0,61( \pm 1,3)^{b}$ \\
\hline \multirow[t]{2}{*}{18} & $+0,30( \pm 3,1)$ & $-0,53( \pm 2,0)^{b}$ & $+0,27( \pm 2,7)$ & $+0,87( \pm 3,4)^{b}$ & $-0,14( \pm 1,3)$ & $-0,43( \pm 1,2)^{b}$ \\
\hline & $\begin{array}{c}c \mathrm{~F}=2,33 \\
p=0,07\end{array}$ & $\begin{array}{c}c F=0,92 ; \\
p=0,42\end{array}$ & $\begin{array}{c}c F=0,92 ; \\
p=0,43\end{array}$ & $\begin{array}{c}c F=0,98 \\
p=0,40\end{array}$ & $\begin{array}{c}c F=1,39 \\
p=0,24\end{array}$ & $\begin{array}{c}c F=0,28 \\
p=0,83\end{array}$ \\
\hline
\end{tabular}

aDiferenças significativas segundo teste $t$ pareado $(p<0,05)$; bDiferenças significativas segundo o teste $t$ não-pareado $(p<0,05)$; Análise de Variância One-Way

Tabela 3

Coeficiente de correlação intraclasse (CCIC)§ entre as medidas referidas e aferidas de peso, estatura e índice de massa corporal (IMC) determinado a partir das medidas referidas. Florianópolis, Santa Catarina 2001.

\begin{tabular}{|c|c|c|c|c|c|c|c|c|c|c|c|c|}
\hline \multirow[t]{3}{*}{ Idade (anos) } & \multicolumn{6}{|c|}{ Peso (kg) } & \multicolumn{2}{|c|}{ Estatura $(\mathrm{cm})$} & \multicolumn{4}{|c|}{ IMC $\left(\mathrm{kg} / \mathrm{m}^{2}\right)$} \\
\hline & \multicolumn{2}{|r|}{ Rapazes } & \multicolumn{2}{|c|}{ Moças } & \multicolumn{2}{|c|}{ Rapazes } & \multicolumn{2}{|c|}{ Moças } & \multicolumn{2}{|c|}{ Rapazes } & \multicolumn{2}{|c|}{ Moças } \\
\hline & $\mathrm{CCIC}$ & IC95\% & $\mathrm{CCIC}$ & IC95\% & $\mathrm{CCIC}$ & IC95\% & $\mathrm{CCIC}$ & IC95\% & $\mathrm{CCIC}$ & IC95\% & $\mathrm{CCIC}$ & IC95\% \\
\hline $15-18$ & 0,96 & $0,95-0,96$ * & 0,96 & $0,95-0,97$ & 0,95 & $0,95-0,96$ & 0,95 & $0,94-0,96$ & 0,93 & $0,92-0,95$ & 0,91 & $0,90-0,95$ \\
\hline 16 & 0,97 & $0,96-0,98$ & 0,96 & $0,94-0,97$ & 0,95 & $0,93-0,96$ & 0,95 & 0,93-0,96 & 0,94 & $0,92-0,96$ & 0,93 & $0,90-0,94$ \\
\hline 17 & 0,98 & $0,97-0,98$ & 0,96 & $0,95-0,97$ & 0,95 & $0,90-0,96$ & 0,95 & $0,92-0,96$ & 0,96 & $0,95-0,97$ & 0,92 & $0,92-0,94$ \\
\hline 18 & 0,97 & $0,96-0,98$ & 0,98 & $0,97-0,99$ & 0,95 & $0,92-0,97$ & 0,92 & $0,91-0,96$ & 0,94 & $0,91-0,97$ & 0,94 & $0,91-0,96$ \\
\hline
\end{tabular}

§Todos os coeficientes de correlação intraclasse são estatisticamente significativos $(p<0,05)$; *IC95\%=Intervalo de confiança $95 \%$.

Apenas $2 \%$ dos rapazes e $3 \%$ das moças relataram a estatura corretamente. Aproximadamente dois terços dos adolescentes (62\% dos rapazes e 59\% das moças) informaram a estatura com diferença de até $2 \mathrm{~cm} ; 86 \%$ e $87 \%$, rapazes e moças respectivamente, com diferença de até $4 \mathrm{~cm} ; 12 \%$ rapazes e $10 \%$ das moças referiram com margem de erro maior que $4 \mathrm{~cm}$ (dados não publicados).

O erro médio do IMC determinado a partir das medidas referidas foi de $-0,07 \mathrm{~kg} / \mathrm{m}^{2}$ nos rapazes ( $p$ $=0,62)$ e $-0,56 \mathrm{~kg} / \mathrm{m}^{2}$ nas moças $(p=0,01)$. As discrepâncias entre o IMC baseado em medidas referidas e aferidas foram estatisticamente mais elevadas nas moças do que nos rapazes $(-0,07 v s-0,56$; $p<0,01)$. Por outro lado, não apresentaram diferenças estatísticas entre as idades estudadas (rapazes $\mathrm{F}=1,39 ; p=0,24$ e moças $\mathrm{F}=0,28 ; p=0,83$ ).
Em $60,7 \%$ dos rapazes e $63,1 \%$ das moças, o IMC determinado com medidas auto-referidas apresentou uma margem de erro de até $1 \mathrm{~kg} / \mathrm{m}^{2} ; 87 \%$ e $86,8 \%$ dos rapazes e moças, respectivamente, com margem de erro de até $2 \mathrm{~kg} / \mathrm{m}^{2}$; pouco mais de $10 \%$ dos jovens ( $11,5 \%$ dos rapazes e $12,4 \%$ das moças) com margem de erro maior que $2 \mathrm{~kg} / \mathrm{m}^{2}$ (dados não publicados).

$\mathrm{O}$ peso auto-relatado apresentou uma concordância elevada com a medida real (rapazes CCIC = 0,96; IC95\%: 0,95-0,96 e moças CCIC = 0,96; IC95\%: 0,94-0,96). Com relação à estatura, a concordância entre as medidas aferidas e referidas foi elevada, e ligeiramente inferior àquela observada para o peso (rapazes CCIC $=0,95$; IC95\%: 0,95-0,96 e moças CCIC =0,95; IC95\%: 0,94-0,96). A concordância entre o IMC calculado a partir das 
Sensibilidade, especificidade e valor preditivo positivo do índice de massa corporal (IMC) determinado a partir das medidas referidas para detectar sobrepeso/obesidade. Florianópolis, Santa Catarina, 2001.

\begin{tabular}{|c|c|c|c|c|c|c|}
\hline \multirow[t]{2}{*}{ Idade (anos) } & \multicolumn{2}{|c|}{ Sensibilidade } & \multicolumn{2}{|c|}{ Especificidade } & \multicolumn{2}{|c|}{ VPP } \\
\hline & Rapazes & Moças & Rapazes & Moças & Rapazes & Moças \\
\hline $15-18$ & 79,1 & 60,0 & 98,4 & 99,2 & 89,8 & 90,0 \\
\hline 15 & 73,3 & 92,3 & 99,0 & 99,2 & 91,7 & 92,3 \\
\hline 16 & 77,3 & 37,5 & 96,1 & 99,2 & 77,3 & 85,7 \\
\hline 18 & 84,6 & 42,9 & 100,0 & 100,0 & 100,0 & 100,0 \\
\hline
\end{tabular}

medidas referidas e o IMC com medidas aferidas, apesar de alta, foi inferior àquela observada para o peso e a estatura, e mais elevada nos rapazes (CCIC $=0,93$; IC95\%: 0,92-0,95) do que nas moças (CCIC $=0,95$; IC95\%: 0,91-0,95). Ocorreram pequenas oscilações no CCIC entre as idades estudadas, nos adolescentes de ambos os sexos. (Tabela 3 ).

Medidas de sensibilidade, especificidade e VPP do IMC determinado a partir das medidas antropométricas auto-referidas, encontram-se descritas na Tabela 4.

A sensibilidade que representa a proporção de adolescentes com sobrepeso/obesidade, corretamente diagnosticados pelo IMC, baseado em medidas aferidas, foi de $71,4 \%$, sendo mais elevada nos rapazes $(79,1 \%)$ em comparação às moças $(60 \%)$. Ao se analisar os índices de sensibilidade, em função da idade dos adolescentes, verificaram-se maiores oscilações nas moças $(37,5 \%$ a $92,3 \%)$ do que nos rapazes $(73,3 \%$ a $84,6 \%)$.

A especificidade que representa a proporção de adolescentes sem sobrepeso/obesidade, corretamente diagnosticados pelo IMC, determinado a partir das medidas auto-referidas, foi de $98,8 \%$, com valores ligeiramente superiores nas moças $(99,2 \%)$ em comparação aos rapazes $(98,4 \%)$. Ao se considerar a idade dos adolescentes, foram verificadas pequenas variações nos níveis de especificidade, principalmente nas moças $(99,2 \%$ a $100 \%)$ em detrimento aos rapazes $(96,1 \%$ a $100 \%)$.

O VPP representa a proporção de adolescentes classificados com sobrepeso/obesidade a partir do IMC, baseado em medidas auto-referidas e que, realmente, apresentam sobrepeso/obesidade; o índice foi de $89,9 \%$ (rapazes $89,8 \%$ e moças $90 \%$ ). O VPP apresentou pequenas oscilações entre as idades estudadas, tanto nos rapazes (94\% a 96\%) quanto nas moças (92\% a 94\%).

\section{Figura 1}

Diferença entre a prevalência de sobrepeso/obesidade determinada a partir do índice de massa corporal (IMC) calculado a partir das medidas referidas vs aferidas. Florianópolis, Santa Catarina, 2001.

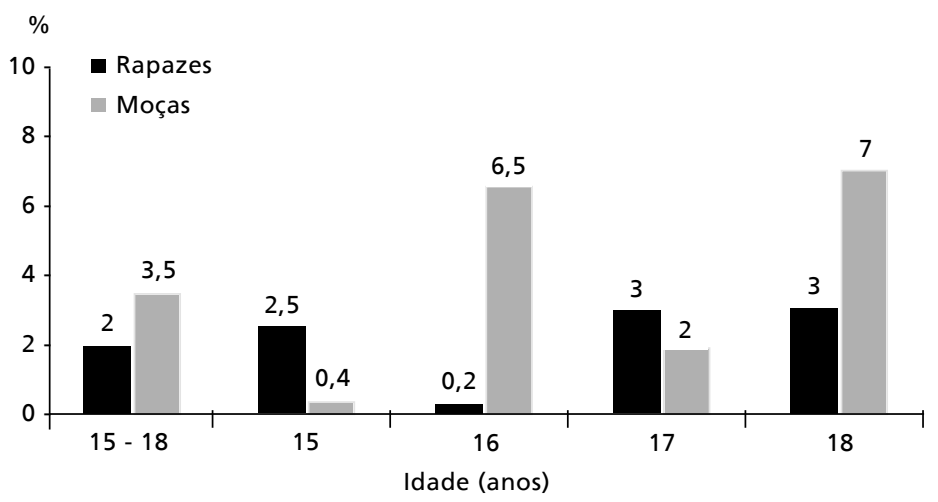

Na Figura 1 estão descritas informações referentes à diferença entre a prevalência de sobrepeso/obesidade determinada a partir do IMC calculado com medidas referidas e o IMC com medidas aferidas. Ao se recorrer ao IMC calculado a partir das informações de peso e estatura referidos pelos adolescentes, verificou-se que a prevalência de sobrepeso/obesidade foi subestimada em 2,6\% ( $p=$ $0,18)$, sendo duas vezes mais elevada nas moças $(3,9 \% ; p<0,01)$ do que nos rapazes $(1,9 \% ; p=$ $0,11)$. Nos rapazes não foram observadas diferenças estatisticamente significativas entre a prevalência de sobrepeso/obesidade determinada a partir das medidas referidas, em comparação as medidas aferidas. Nas moças, aos 16 e 17 anos, 6,5\% e 7\%, respectivamente, a prevalência de sobrepeso/obesidade determinada a partir das medidas referidas apresentou valores estatisticamente inferiores aos observada nas medidas aferidas. 


\section{Discussão}

Ao informarem suas medidas antropométricas, os adolescentes tendem a subestimar o peso e superestimar a estatura e, por conseguinte, o IMC determinado a partir dessas medidas. As discrepâncias observadas entre as medidas aferidas e referidas foram pequenas, principalmente nos rapazes em comparação as moças. Uma proporção elevada de adolescentes $(>85 \%)$ referiu o peso $(+/-5 \mathrm{~kg})$ e a estatura $(+/-4 \mathrm{~cm})$ com uma margem de erro relativamente pequena. Como conseqüência, o IMC baseado nessas medidas também apresentou uma margem de erro pequena $\left(+/-2 \mathrm{~kg} / \mathrm{m}^{2}\right)$.

Esses resultados se assemelham aos achados de estudos anteriores que também evidenciaram superestimativa na estatura e subestimativa no peso e no IMC, determinado a partir dessas medidas, principalmente nas moças, $6,8,16,17$ e divergem, em parte, de outros que observaram maior superestimativa na estatura $9,11,18$ ou no peso 5,7 entre os rapazes.

A precisão com que os adolescentes auto-relatam o peso, a estatura e, por conseguinte, o IMC baseado nessas medidas, não se mostrou influenciada pela idade dos adolescentes. Esses resultados foram diferentes dos apresentados no estudo de Himes et al.,16 com adolescentes de 12 a 19 anos, que apontou tendência a maior precisão no auto-relato do peso, estatura e IMC com o avanço da idade. Diferenças quanto à faixa etária da população envolvida em cada estudo podem explicar, em parte, as divergências encontradas, tendo em vista que as maiores diferenças entre as medidas aferidas e referidas parecem ocorrer nos adolescentes mais jovens (12 a 15 anos). 16

As discrepâncias encontradas entre as medidas auto-referidas e aferidas podem ser decorrentes de diversas fontes. Nos estudos de validação das medidas auto-referidas, as medidas aferidas, que serão o "padrão ouro", são realizadas por um pesquisador, seguindo padronização específica e utilizando equipamentos adequados (especializados e calibrados). Já as medidas referidas, na maioria das vezes, são realizadas pelos próprios adolescentes, adotando procedimentos inadequados (uso de roupas pesadas, calçados, portando objetos), utilizando instrumentos inadequados e em períodos distintos.

Outro aspecto a ser considerado, é que os adolescentes podem estar aferindo com baixa freqüência suas medidas antropométricas e que, combinado ao rápido processo de crescimento, passam a ter uma informação desatualizada sobre o valor real do seu peso e da sua estatura. Dessa forma, uma larga parcela da população jovem pode não conhecer a medida real do seu peso e estatura. Adolescentes em estágio púbere e pós-púbere 10 e os que têm o peso e a estatura freqüentemente aferidos, ${ }^{19}$ auto-relatam com maior precisão essas medidas.

Por fim, a preocupação excessiva dos jovens com a imagem corporal e o culto ao "corpo perfeito", tende a levar os adolescentes a informarem o peso e a estatura baseados em medidas que gostariam de ter, o que parece ser reforçado pelos elevados índices de insatisfação com a imagem corporal observados em adolescentes. ${ }^{20}$

No presente estudo, a concordância entre as medidas referidas e aferidas foi alta $(\mathrm{CCIC}<0,90)$, nos adolescentes de ambos os sexos e de diferentes idades (15 a 18 anos). Esses resultados, apesar de ligeiramente superiores, estão de acordo com a literatura.6,16 Outras investigações com adolescentes, que recorreram à estatística $r$ de Pearson, também descreveram correlações elevadas entre as medidas auto-referidas e aferidas, com pequenas variações por sexo e idade dos adolescentes.5,7,8,17

Os índices de sensibilidade do IMC baseados em medidas referidas, para detectar sobrepeso/obesidade, no presente estudo foram elevados nos rapazes $(79,1 \%)$ e baixos nas moças $(60 \%)$. Esses resultados são semelhantes aos descritos por Davis e Gergen, 7 $76 \%$ nos rapazes e $59 \%$ nas moças, e Brener et al.,8 $60 \%$ nos adolescentes de ambos os sexos. Por outro lado, Galán et al., 5 em estudo com adolescentes de Madrid, revelou baixos níveis de sensibilidade para o IMC baseado em medidas referidas (IMC maior que percentil 85$)$ tanto nos rapazes $(54,7 \%)$ quanto nas moças $(56,3 \%)$. Desta forma, ao se recorrer às medidas informadas pelos adolescentes, aproximadamente dois em cada dez rapazes e quatro em cada dez moças com sobrepeso/obesidade, deixaram de ser classificados como tal.

O comportamento dos valores de especificidade do IMC, determinado com base nas medidas referidas, encontrados nos adolescentes de Florianópolis, foi similar ao que tem sido observado em outras investigações: valores elevados (> 95\%) e ligeiramente superiores nas moças em comparação aos rapazes. 5,7 O IMC determinado a partir de medidas referidas demonstrou elevada capacidade de diagnosticar corretamente aqueles adolescentes que não apresentam sobrepeso/obesidade. Cerca de nove em cada dez adolescentes sem sobrepeso/obesidade foram corretamente classificados pelo IMC determinado pelas medidas referidas de peso e estatura.

Os índices do VPP (rapazes 89,8\% e moças 90\%) foram superiores aos observados no estudo de Galán et al.,5 $83 \%$ nos rapazes e 85,9\% nas moças, e inferiores aos descritos por Brener et al.,8 $96 \%$ nos 
adolescentes de ambos os sexos. Entre os adolescentes classificados com sobrepeso/obesidade pelo IMC, determinado por medidas auto-referidas pelos adolescentes, nove em cada dez realmente apresentaram tal condição.

O viés das medidas auto-referidas pelos adolescentes também foi analisado, comparando-se a prevalência de sobrepeso/obesidade determinada a partir do IMC, determinado com base nas medidas referidas $v s$ medidas aferidas. A prevalência de sobrepeso/obesidade foi subestimada em 2,6\% (IMC medidas aferidas $=10,3 \%$ vs IMC medidas referidas $=12,9 \%$ ) quando se recorreu às medidas referidas, entretanto, essa diferença não foi estaticamente significativa. O uso de medidas referidas subestimou a prevalência de sobrepeso/obesidade em 1,9\% nos rapazes $(p>0,05)$ e $3,5 \%$ nas moças $(p=0,02)$.

A subestimativa na prevalência de sobrepeso/obesidade pelo IMC, determinado com medidas referidas observadas na população investigada, foi inferior à observada em alguns estudos, $8,16,18,20$ similar à encontrada por Crawley e Portides, 11 e superior à relatada por Fortenberry. 9

Como se trata de um estudo de base escolar, a extrapolação dos resultados para a população de adolescentes em geral fica comprometida, por ter incluído somente os adolescentes que estavam freqüentando as séries do ensino médio e que estavam em sala de aula no dia da coleta de dados. Uma outra limitação deste estudo é que não foi perguntado aos adolescentes o tempo transcorrido e as condições sob as quais foram realizadas as medidas de peso e estatura referidas pelos mesmos.
Ao serem analisadas como variáveis contínuas, o peso, a estatura e o IMC baseado nessas medidas, apresentaram um erro médio pequeno e alta concordância com as respectivas medidas aferidas. Quando utilizadas na avaliação do estado nutricional a partir do IMC, as medidas auto-referidas mostraram uma boa capacidade de discriminar os adolescentes com sobrepeso/obesidade e elevado poder de discriminar os adolescentes sem sobrepeso/obesidade e, conseqüentemente, estimar a prevalência de sobrepeso/obesidade com pequena margem de erro $(<2 \%)$.

Entretanto, previamente à utilização em grande escala, recomenda-se investigar, em uma subamostra da população alvo, a precisão com que os adolescentes referem o peso e a estatura, e identificar as características associadas com erros no auto-relato dessas medidas. Esse procedimento pode favorecer a determinação de fatores de correção para as medidas referidas, particularmente, no IMC calculado a partir da utilização dessas medidas. Esse procedimento, quando necessário, tem possibilitado um aumento na precisão da classificação do estado nutricional dos adolescentes. 17

Desse modo, essas informações representam medidas válidas como forma de aproximação aos valores mensurados, podendo ser utilizadas, em substituição às medidas aferidas, para o diagnóstico do estado nutricional de adolescentes a partir do IMC, em estudos epidemiológicos, principalmente quando a economia de recursos, a simplificação do trabalho de campo e a abrangência do levantamento populacional forem aspectos decisivos.

\section{Referências}

1. Chu NF, Rimm EB, Wang DJ, Liou HS, Shieh SM. Clustering of cardiovascular disease risk factors among obese schoolchildren: the Taipei Children Heart Study. Am J Clin Nutr. 1998; 67: 1141-6.

2. Williams DP, Scott BG, Lohman TG, Harsha DW, Srinivasan SR, Webber LS, Berenson GS. Body fatness and risk for elevated blood pressure, total cholesterol, and serum lipoprotein rations in children and adolescents. Am J Public Health. 1992; 82: 358-63.

3. CDC (Center for Disease Control and Prevention). Youth risk behavior surveillance, United State - 2003. Surveillance summaries. MMWR. 2004: 53 (No. SS-2).

4. HEA (Health Education Authority). Today's young adults: 16-19 year olds look at diet, alcohol, drugs and sexual behavior. London; 1992.

5. Galán I, Gandarillas A, Febrel C, Meseguer CM. Validación del peso y talla autodeclarados en población adolescente. Gac Sanit. 2001; 15: 490-7.
6. Strauss RS. Comparison of measured and self-reported weight and height in a cross-sectional sample of young adolescents. Int J Obes. 1999; 23: 904-8.

7. Davis H, Gergen JP. The weights and heights of MexicanAmerican adolescents: the accuracy of self-reports. Am J Public Health. 1994; 84: 459-62.

8. Brener ND, McManus T, Galuska DA, Lowry R, Wechsler H. Reliability and validity of self-reported height and weight among high school students. J Adolesc Health. 2003; 32: 281-7.

9. Fortenberry D. Reliability of adolescent's report of height and weight. J Adolesc Health. 1992; 13: 114-7.

10. Abraham S, Luscombe G, Boyd C, Olesen I. Predictors of the accuracy of self-reported height and weight in adolescent female school students. Int J Eat Disord. 2004; 36: 7682. 
11. Crawley HF, Portides G. Self-reported versus measured height, weight and body mass index amongst 16-17 year old British teenagers. Int J Obes. 1995; 19: 579-84.

12. Silveira EA, Araújo CL, Gigante DP, Barros AJD, Lima MS. Validação do peso e altura referidos para o diagnóstico do estado nutricional em uma população de adultos do Sul do Brasil. Cad Saúde Pública. 2005; 21: 235-45.

13. Fonseca MJM, Faerstein E, Chor D, Lopes CS. Validade de peso e estatura informados e índice de massa corporal: estudo pró-saúde. Rev Saúde Pública. 2004; 38: 392-8.

14. Gordon, CC, Chumlea, WC, Roche, AF. Stature recumbert, lenght, and weight. In: Lohman, TG, Roche, AF, Martorell R. Anthopometric standardization reference manual. Champaingn, Illinois, Human Kinetics; 1988. p. 3-8.

15. Cole TJ, Bellizzi MC, Flegal KM, Dietz WH. Establishing a standard definition for child overweight and obesity worldwide: international survey. BMJ. 2000; 320: 1-6.

16. Himes JH, Hannan P, Wall M, Neumark-Sztainer D. Factors associated with errors in self-reports of stature, weight, and body mass index in Minnesota Adolescents. Ann Epidemiol. 2005; 15: 272-8.

Recebido em 18 de outubro de 2006

Versão final apresentada em 12 de fevereiro de 2007

Aprovado em 14 de março de 2007
17. Giacchi M, Mattei R, Rossi S. Correction of the selfreported BMI in a teenage population. Int J Obes. 1998; 22: 673-7.

18. Wang Z, Patterson CM, Hills AP. A comparison of selfreported and measured height, weight and BMI in Australian adolescents. Aust N Z Public Health. 2002; 26 : 473-8.

19. Page RM, Lee C, Miao N. Assessing prevalence of overweight and obesity through self-reports of height and weight by high school students in Taipei, Taiwan. J Sch Health. 2004; 74: 401-7.

20. Rovira RF, Pons IF, Martínez MIM, Sánchez RR. Selfreported versus measured height, weight and body mass index in Spanish Mediterranean teenagers: effects of gender, age and weight on perceptual measures of body image. Ann Nutr Metab. 2002; 46: 68-72. 